\title{
Examination of Orbital Tissues in Murine Models of Graves' Disease Reveals Expression of UCP-1 and the TSHR in Retrobulbar Adipose Tissues
}

\author{
Authors \\ K. T. M. Johnson ${ }^{1}$, B. Wiesweg ${ }^{1}$, M. Schott' ${ }^{2}$, M. Ehlers ${ }^{2}$, M. Müller ${ }^{1}$, W. B. Minich ${ }^{3}$, Y. Nagayama ${ }^{4}$, \\ E. Gulbins ${ }^{5}$, A. K. Eckstein ${ }^{1}$, U. Berchner-Pfannschmidt \\ Affiliations \\ Affiliation addresses are listed at the end of the article
}

\author{
Key words \\ - Graves' orbitopathy \\ - animal model \\ - brown adipose tissue \\ - macrophages
}

received 04.06 .2012

accepted 10.12.2012

Bibliography DOI http://dx.doi.org/

10.1055/s-0032-1333224

Published online:

February 5, 2013

Horm Metab Res 2013;

45: 401-407

(c) Georg Thieme Verlag KG

Stuttgart · New York

ISSN 0018-5043

Correspondence

Prof. Dr. med. A. K. Eckstein

Department of Ophthalmology

University of Duisburg-Essen

45147 Essen

Germany

Tel.: + 49/201/7232907

Fax: +49/201/723 5641

anja.eckstein@uk-essen.de

\section{Abstract \\ $\nabla$}

Over the past decade a number of murine models of Graves' disease (GD) have been described. The full symptom complex, including typical orbital changes, however, could not yet be induced. In this report, we examined the influence of modified immunization protocols on orbital pathology. C57BL/6 and BALB/c mice were immunized against the human TSH receptor (TSHR), using either a TSHR encoding plasmid or a TSHR A-subunit adenovirus. Prior to immunization with the TSHR plasmid, regulatory $\mathrm{T}$ cells were depleted in one group of each strain. TSHR-stimulating antibodies (TSAbs) were evaluated and orbits were stained immunohistochemically for $\mathrm{F} 4 / 80$, uncoupling protein-1 (UCP-1) and the TSHR. We

\section{Introduction}

$\nabla$

Graves' disease (GD) is a systemic autoimmune disease, which is comprised of hyperthyroidism, orbitopathy, and, more rarely, dermopathy and acropachy $[1,2]$. Twenty-five to $50 \%$ of patients, suffering from GD, show symptoms of orbital inflammation $[1,3]$. Clinical symptoms of orbitopathy are due to inflammation of orbital connective tissue, inflammation, and fibrosis of the extraocular muscles and adipogenesis. Hyperthyroidism is caused by stimulating TSH receptor antibodies (TSAb). The receptor is also regarded as one of the major antigens in the orbit. Clinically, high TSAb concentrations are associated with a higher prevalence and more severe course of extra-thyroidal symptoms, especially orbitopathy [4-7].

While there is no spontaneous animal model for $\mathrm{GD}$, a number of induced mouse models have been described over the past decades. All of these models have in common that by immunization against the hTSHR or parts thereof an autoim- found that after depletion of regulatory T cells, incidence of TSAb was increased in TSHR plasmid immunized C57BL/6 mice. Examination of early immunized mice showed no antibody production. However, a TSHR epitope-specific cellular immune response could be detected by tetrameranalyses. Adenoviral immunization lead to TSAb production in all but one animal. Analysis of F4/80 positive cells in retrobulbar fat revealed no significant macrophage infiltration in the orbits of immunized mice. Immunohistochemical staining shows co-localization of F4/80 positive cells, UCP-1 and the TSHR in retrobulbar fat. Though targets for TSHR autoimmunity could clearly be shown, immunization methods were not efficient enough to cause clear signs of orbital inflammation.

mune reaction is induced. This can be facilitated using a variety of techniques, such as introduction of the TSHR as a recombinant protein, of foreign cells expressing the receptor, or via genetic immunization with cDNA, or adenoviral constructs expressing the extracellular domain of the hTSHR or its A-subunit [8-17]. Immunizations against the murine TSHR have failed to result in desired autoimmunity, due to the host organism's tolerance [18]. While thyroid autoimmunity and hyperthyroidism can be reliably induced using adenovirus encoding for the TSHR A-subunit $[19,20]$ or through intramuscular electroporation of TSHR encoding plasmids [21], orbital manifestations had been reported by only one group of investigators, describing infiltration of orbital tissues by macrophages and lymphocytes [22]. The reported orbital manifestations could not be reproduced by other investigators $[23,24]$. Sporadic examination of extraocular muscles by other groups showed no evidence of eye muscle inflammation or edema $[14,16]$. Additionally, the process of eye muscle 
Table 1 Immunization schemes for C57BL/6 and BALB/C mice.

\begin{tabular}{lllll} 
Strain & Immunogen & Immunizations & + anti-CD25 & Mice, $\mathbf{n}$ \\
C57BL/6 & D633HTSHR & $4 \times$ & - & 10 \\
\hline C57BL/6 & D633HTSHR & $4 \times$ & + & 10 \\
C57BL/6 & AdTSHR289 & $2 \times$ & - & 6 \\
\hline BALB/C & D633HTSHR & $4 \times$ & - & 6 \\
BALB/C & D633HTSHR & $4 \times$ & + & 6 \\
\hline BALB/C & AdTSHR289 & $2 \times$ & - & 6 \\
\hline
\end{tabular}

Female 4-6 week old C57BL/6 and BALB/c mice were immunized 4 times in 2 week intervals against the $\mathrm{D} 633 \mathrm{H}$ activating mutant of the human TSHR with and without depletion of CD25 + T-cells. Two additional groups of each strain were immunized against the A-Subunit of the human TSHR using a recombinant adenovirus (AdTSHR289). Immunization of mice with AdTSHR289 was performed in Nagasaki

preparation was not described in detail. Since the process of removing extraocular muscles from the orbit, even if performed with the utmost care, may have led to tissue strain and also bleeding, artefact formation may not be excluded. The aim of our study was to establish a more refined technique to reliably examine mouse orbits without the risk of artefact induction and to examine the influence of modified immunization protocols on orbital pathology.

\section{Materials and Methods}

\section{Mice and immunization protocols}

For anatomic and histologic examination, female C57BL/6 and BALB/c mice, as well as C3H, A/J, FrBn, and NMRI (4-5 weeks old) were originally purchased from Harlan Laboratories (Rossdorf, Germany) and kept in a specific pathogen-free facility. Attempts to induce Graves' disease were performed on 6 groups of C57BL/6 and BALB/c mice, which were immunized using either TSHR cDNA or the AdTSHR289 construct. Mice of the same strains, sex, and age were used as controls. Immunization with AdTSHR289 was performed in Nagasaki. A different set of controls was used for mice that were raised in Essen and Nagasaki, respectively, to avoid influence of environmental factors.

Experiences of 3 previously described animal models were combined in our approach as detailed in $\bullet$ Table 1. Genetic immunization against the $\mathrm{D} 633 \mathrm{H}$ activating mutant of the human TSHR, as described by Ho et al. [25], was combined with depletion of CD25 + regulatory T-cells as described by Saitoh and Nagayama [17]. Prior to injection in the M. quadriceps femoris, $100 \mu \mathrm{g}$ plasmid was adsorbed to an aluminum phosphate adjuvant (AdjuPhos, Brenntag Biosector, Frederiksund, Denmark) to facilitate stronger immune reactions [26]. Injection volumes per site were $50 \mu \mathrm{l}$. Immunization with the AdTSHR289 construct was performed as described previously [17]. To evaluate immunization, animals were sacrificed, blood was collected by cardiac puncture under anesthesia with $10 \mu \mathrm{l}$ i.p./g (body weight) ketamine $2 \% / x y l a z i n e ~ 0.1 \%$ (CEVA Tiergesundheit GmbH, Düsseldorf, Germany); thyroid and orbits were collected and prepared for histologic examination. Animals were kept under standard conditions and were fed regular chow (ssniff $M-Z$, ssniff Spezialdiäten $\mathrm{GmbH}$, Germany). All procedures performed on mice in Essen were approved by the State Office for Nature, Environment and Consumer Protection of North Rhine-Westphalia, Germany in accordance with $\S 8$ of the Animal Protection Act (Tierschutzgesetz) in the edition of $5 / 18 / 2006$. All procedures performed on mice in Nagasaki were performed in accordance with the Guideline for Animal Experimentation of Nagasaki University with approval of the Institutional Animal Care and Use Committee. Two animals, one from the undepleted C57BL/6 and one from the depleted group had to be euthanized before examination, due to hydrocephalus and malformed teeth, respectively.

\section{Plasmid and adenovirus preparation}

The activating mutant D633H - a kind gift from Dr. Su-Chin Ho of SingHealth Research, Singapore - was introduced via PCR into the wild type full length sequence for the human TSHR as described previously [25]. Construction and purification of adenovirus containing amino acid residues 1-289 of the human TSHR (TSHR-289-ad) has also been described previously [17,19]. The vector was provided by Drs. B. Rapoport and S. M. McLachlan (Cedars-Sinai Medical Center, University of California, Los Angeles, CA, USA). Adenovirus was propagated in and purified by $\mathrm{CsCl}$ density gradient centrifugation [17].

\section{Antibody preparation}

Rat anti-mouse CD25 was obtained through purification of hybridoma supernatants [27]. Antibody functionality was tested through FACS analysis of CD4+CD8+CD25+ (FITC-conjugated anti-mouse CD4, PE-conjugated anti-mouse CD8, APC-conjugated anti-mouse CD25, BD Pharmingen, Heidelberg, Germany) mouse splenocyte population 4 days after i.p. injection.

\section{Tetramer analysis}

After exsanguination - but before perfusion fixation - mice were splenectomized. Spleens were crushed and homogenized in unsupplemented DMEM. Splenocytes were isolated using Histopaque (Sigma-Aldrich, St. Gallen, Switzerland) according to a standardized protocol, resuspended and washed in DMEM, supplemented with $10 \% \mathrm{FCS}$. Cells were then stored at $-80^{\circ} \mathrm{C}$ so they could be analyzed simultaneously.

For tetramer analysis, cells were thawed and resuspended in $100 \mu \mathrm{l}$ MACS buffer (Miltenyi, Bergisch-Gladbach, Germany), supplemented with $50 \mu \mathrm{l}$ FCS, $10 \mu \mathrm{l}$ FCR blocking reagent (Miltenyi, Bergisch-Gladbach, Germany) and $4 \mu$ l tetramer (Glycotope Biotechnology, Heidelberg, Germany), followed by incubation on ice for $1 \mathrm{~h}$. All TSHR tetramers and corresponding peptides were selected according to their calculated binding scores calculated with SYFPEITHI (www.syfpeithi.de), a database of more than 7000 peptide sequences known to bind MHC class I and class II molecules. Binding scores of $\geq 15$ were considered highly immunogenic (reviewed in [28]). Based on the amino acid sequence of human TSHR, all tetramers (H2-Kb specific) had high binding scores between 22 and 24 . The following PE-conjugated tetramers and corresponding epitopes were chosen: T1 [amino acid position (AA) 412-419]: MGYKFLRI, T2 (AA68-73): PSHAFSNL, T3 (AA93-100): ESHSFYNL. Anti-CD8 FITC was added to each of the samples besides anti-IgG1 FITC and PE and anti-CD45 FITC antibodies, which were used as a negative and positive control, respectively. For additional negative control the tetramer "SIINFEKL" was used, delivering only a positive signal for animals immunized with ovalbumin. Cells were analyzed on a FACS Calibur (BD Bioscience, San Jose, CA, USA) equipped with BD-CellQuest-Pro software.

\section{TSAb measurements}

Mice were exsanguinated by cardiac puncture under anesthesia with ketamine/xylazine. Whole blood was kept on ice and spun down at 6000 RPM (Mikro 200R Hettich Centrifuges, Tuttlingen, Germany) for $15 \mathrm{~min}$. After collection, serum was stored at 


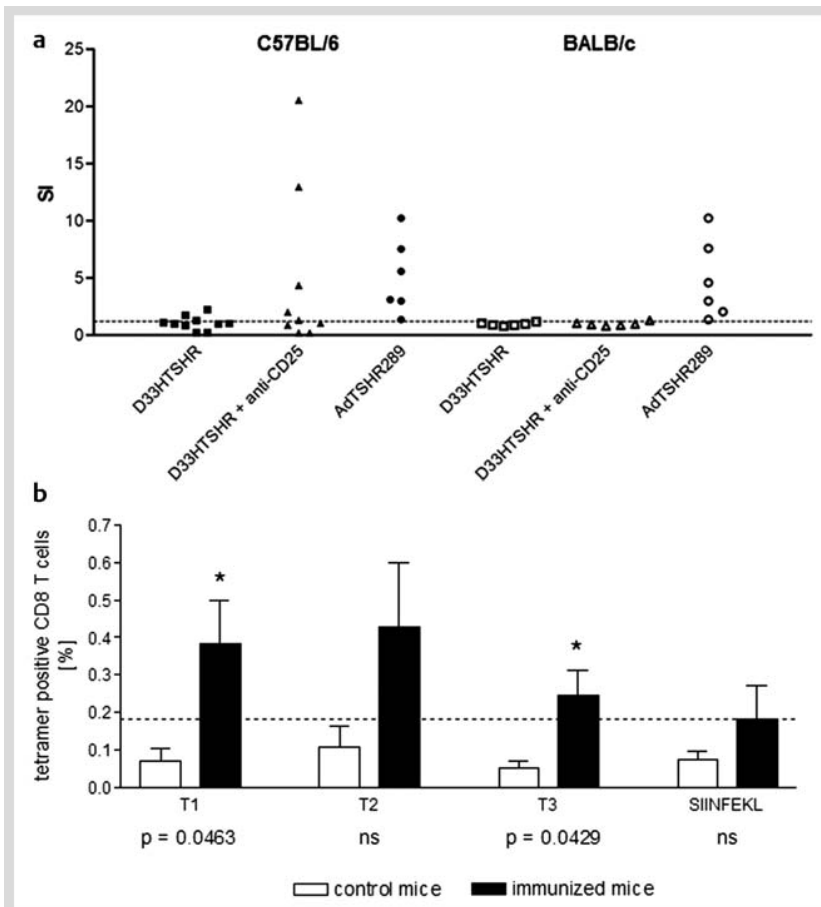

Fig. 1 a TSAb in D633HTSHR and AdTSHR289 immunized mice. Stimulation indices (SI) of serum TSHR antibodies (TSAbs) in immunized C57BL/6 (black symbols) and BALB/C (white symbols) mice, immunized as described in 1 Table 1 . The dotted line represents the $95 \% \mathrm{Cl}$ of unimmunized controls. TSAbs were measured as described in the methods section. Thyroid stimulation index (SI) was calculated as:

$\mathrm{SI}=\frac{\text { rlu }}{\mathrm{s}}($ immunized $) / \frac{\text { rlu }}{\mathrm{s}}$ (control)

immunized mice/rlu/s euthyroid control mice. b Tetramer Analysis reveals an early TSHR-specific immuneresponse. Immunization of C57BI/6 mice with D633HTSHR resulted in a significant induction of CD8 positive $T$ cells recognizing the TSHR-epitopes MGYKFLRI (AA 412-419) and ESHSFYNL (AA 93-100), that were identified using tetramer 1 and 2 (T1, T2). Immunization led to the development of $0.34 \% \mathrm{~T} 1$ - and $0.22 \% \mathrm{~T} 3$-tetramer positive CD8 positive T cells in comparison to $0.07 \%$ and $0.05 \%$ specific $T$ cells in the untreated group (T1: $p=0.0463 ; T 3: p=0.0429)$. The mean values including the SEM are shown for $n=3$ mice in each group. The dotted line indicates the background staining measured by using the SIINFEKL tetramer.

$-80^{\circ} \mathrm{C}$ until measurement of TSAbs was performed. TSAbs were measured as described by Morgenthaler et al. [29]. Thyroid stimulation index (SI) was calculated as:

$\mathrm{SI}=\frac{\mathrm{rlu}}{\mathrm{S}}($ immunized $) / \frac{\mathrm{rlu}}{\mathrm{S}}($ control $)$

\section{Histology}

Mice were anesthesized as described above. Perfusion fixation was performed as previously described [30]. The orbit was removed completely, including adjacent bony structures, to leave its contents untouched. Orbital tissues were decalcified in EDTA for $24 \mathrm{~h}$. For HE staining single slides of paraffin embedded tissue were rehydrated and stained with Meyers hemalum and eosin (Chroma 1B, Schmid GmbH, Münster, Germany) for 3 min each. Immunohistochemical visualization of macrophages was performed using rat-anti-mouse F4/80 (1:100, Abd Serotech, Oxford, UK). Uncoupling protein-1 (UCP-1) was stained using rabbit anti-mouse UCP-1 antibody (1:200, Alpha Diagnostic International, San Antonio, TX, USA) and TSHR was stained using a rabbit anti-human TSHR antibody (1:200, MBL Int., Woburn, MA, USA). The embedded tissues were incubated overnight at $4{ }^{\circ} \mathrm{C}$ with primary antibodies. The biotin coupled secondary antibody (biotin goat-anti-rat or anti-rabbit, Santa Cruz Biotech Inc.) was visualized using commercially available $A B C$ and $D A B-$ Kits (Vectastain ABC/DAB, Vector Labs, Burlingame, CA, USA). Endogenous peroxidase activity was inhibited with $3 \% \mathrm{H}_{2} \mathrm{O}_{2}$. Slides were counterstained with hematoxylin for 90 s. F4/80 positive cell counts were established blindly in duplicate at 400fold magnification using a Nikon eclipse E1000 microscope.

\section{Statistical analysis}

Statistical analysis was performed using GraphPad Prism 5.0 (GraphPad Software Inc., San Diego CA). Data was analyzed using the Kruskal-Wallis test; post-testing was conducted with Dunn's multiple comparison test.

\section{Results \\ $\nabla$}

\section{Anti-TSHR antibodies}

Measured TSAb stimulation indices are displayed in $\bullet$ Fig. 1. Two out of 10 mice in the C57BL/6 group, immunized with D633HTSH showed low TSAb stimulation indices (mouse 2: 1.76 and mouse 3: 2.23) ( $\odot$ Fig. 1a first row). None of the other animals showed any relevant serum activity of TSAb. Depletion of $\mathrm{CD} 25$ + regulatory $\mathrm{T}$ cells resulted in a significant higher incidence of stimulating antibodies ( 4 out of 9 mice) and stimulation indices were markedly higher (mice 2: 20.55, 3: 12.95, 4: 4.35, and 6: 2.0) (๑ Fig. 1a second row), though mean stimulation indices of both groups did not differ significantly. Likewise, none of the plasmid immunized BALB/c mice (depleted or undepleted) showed activities of TSAb outside the $95 \% \mathrm{CI}$ of controls ( $\bullet$ Fig. 1a fourth and fifth rows). In contrast, all AdTSHR289 immunized mice of the C57BL/6 and 5 out of $6 \mathrm{BALB} / \mathrm{c}$ showed significantly more frequent $(\mathrm{p}=0.012)$ and higher $(\mathrm{p}<0.05)$ activities of TSAbs than plasmid treated mice of the respective strains. Stimulation indices varied between 1.35 and 10.22. There were no significant differences between BALB/C and C57BL/6 mice.

\section{Tetramer analysis}

The low incidence of stimulating TSAbs in D633HTSHR immunized mice led us to perform tetramer analyses to detect specific TSHR immune reactions during the early phase of immunization. TSHR specific $\mathrm{T}$ splenocytes were quantified in C57BL/6 mice undergoing a shorter immunization protocol with 1-3 × D633HTSHR. A significant proportion of CD8 + positive T-cells presented anti-TSHR responsiveness as indicated in $\bullet$ Fig. 1b. Using tetramer 1 and 3 to detect $\mathrm{T}$ cells identifying TSHR at AA positions MGYKFLRI (412-419) and ESHSFYNL (AA93-100), a significant increase in TSHR-specific CD8 positive $\mathrm{T}$ cells was observed ( $\bullet$ Fig. 1b). In detail, following immunization the frequency of target-specific $\mathrm{T}$ cells increased markedly to $0.34 \%$ for $\mathrm{T} 1$ ( $\mathrm{p}=0.0463), 0.38 \%$ for $\mathrm{T} 2$, and $0.22 \%(\mathrm{p}=0.0429)$ for $\mathrm{T} 3$ in comparison to untreated mice $(0.07 \%, 0.11 \%$, and $0.05 \%$, respectively). 

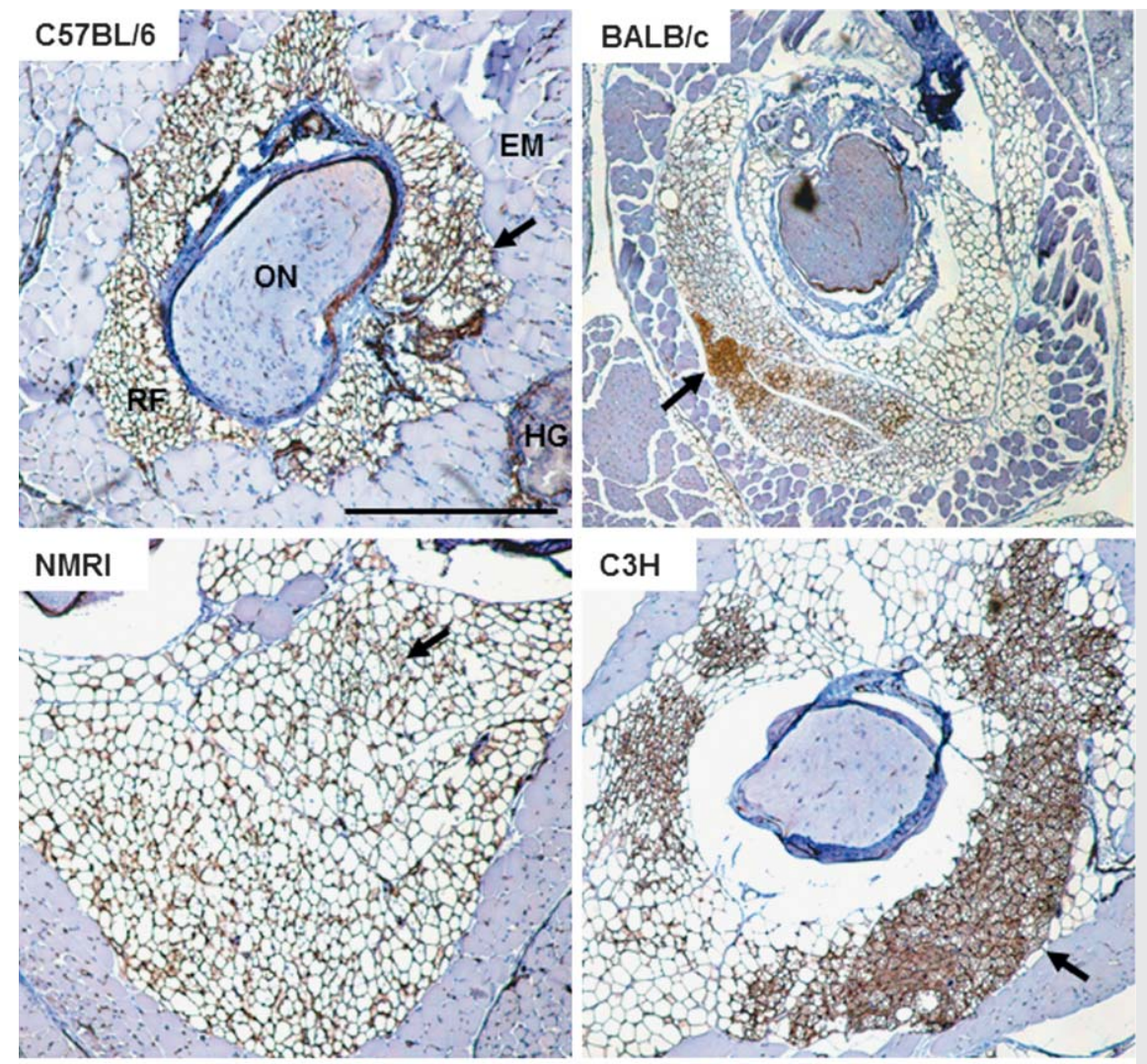

Fig. 2 Immunohistochemical visualization of mouse orbits. To characterize the retrobulbar fat, orbits of 6 different strains (C57BL/6, BALB/c, $\mathrm{NMRI}, \mathrm{C} 3 \mathrm{H}, \mathrm{FrBn}, \mathrm{A} / \mathrm{J}$ ) were stained immunohistichemically, using UCP-1 as a marker for brown fat. Images were captured at $100 \times$ magnification. ON: optical nerve; RF: retrobulbar fat; EM: extraocular muscles; HG: Harderian gland. Arrows indicate smaller vacuoles containing adipose tissues intensively stained with UCP-1. Bar represents $100 \mu \mathrm{m}$.
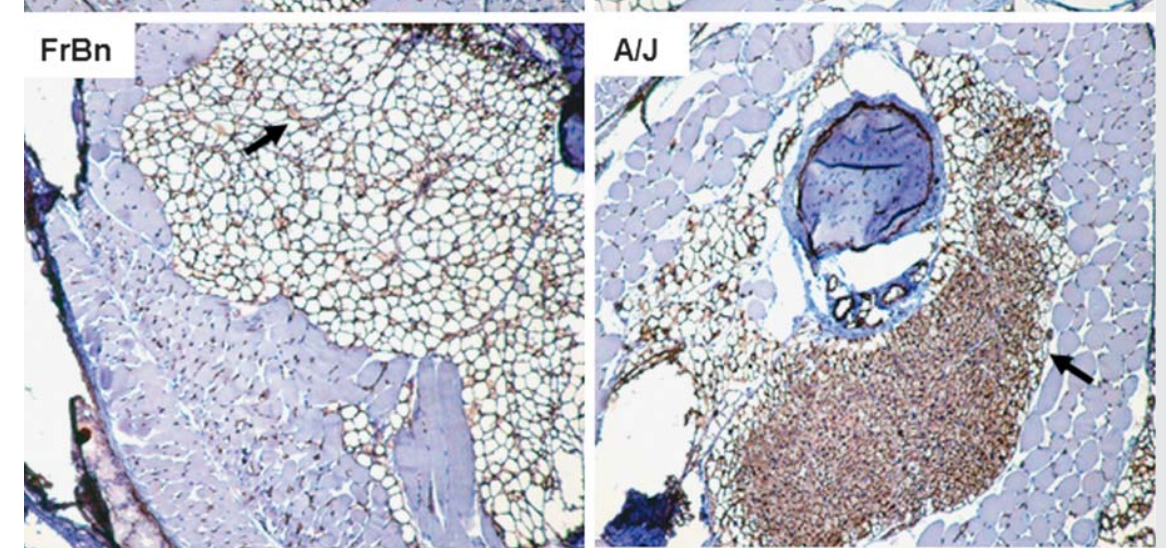

Histologic preparation of orbital tissues and target antigens

To exclude preparation artefacts we developed a technique of in toto preparation of orbits. In $\odot$ Fig. 2, UCP-1 staining of slices of several mice strains is displayed. Clear anatomical landmarks can be recognized. The centrally located optic nerve is surrounded by orbital fat and embedded in the muscle cone. UCP-1 as marker for brown adipose tissue was concentrated in the smaller vacuoles of adipose tissue ( $\boldsymbol{\theta}$ Fig. 2 , arrows). The target antigen TSHR could also be clearly detected in the orbital fat of all investigated strains ( $\bullet$ Fig. 3 ).

\section{Inflammatory cells in the orbital tissues}

Valued Prevalence of immune cells was low in the orbital fat of all mice (a representative example is shown in $\odot$ Fig. 3). Quantitative analysis of cell counts is displayed for $\mathrm{F} 4 / 80$ positive cells (macrophages) in 0 Fig. 4. No infiltration of macrophages was seen in the D633HTSHR immunized C57BL/6 ( $\odot$ Fig. 4a) or BALB/c ( $\odot$ Fig. 4b) nor in AdTSHR289 ( $\odot$ Fig. 4c) immunized $\mathrm{C} 57 \mathrm{BL} / 6$ or $\mathrm{BALB} / \mathrm{c}$ when compared to controls. Comparing groups as a whole yielded no significant differences. Overall macrophage counts were lower in BALB/c mice when compared to $\mathrm{C} 57 \mathrm{BL} / 6$. Because the Harderian gland, which is found only in vertebrates that possess a nictitating membrane, was suggested to be a location of immune response [31], we decided to establish F4/80 cell counts in the Harderian glands of AdTSHR289 immunized C57BL/6. Overall we found more F4/80 positive cells in the Harderian gland tissues than in the orbital fat; however, there were no significant differences in macrophage counts between immunized $(20.53 \pm 1.69$ SEM F4/80 pos. cells/VF) and control mice (22.49 \pm 2.61 SEM F4/80 pos. cells/VF).

\section{Discussion}

In the present study, we describe a technique to reliably examine mouse orbits. The new technique was applied to examine the influence of modified immunization protocols against the hTSHR to induce Graves' orbitopathy like changes in the murine orbit. Modified immunization protocols lead to induction of TSAb tit- 


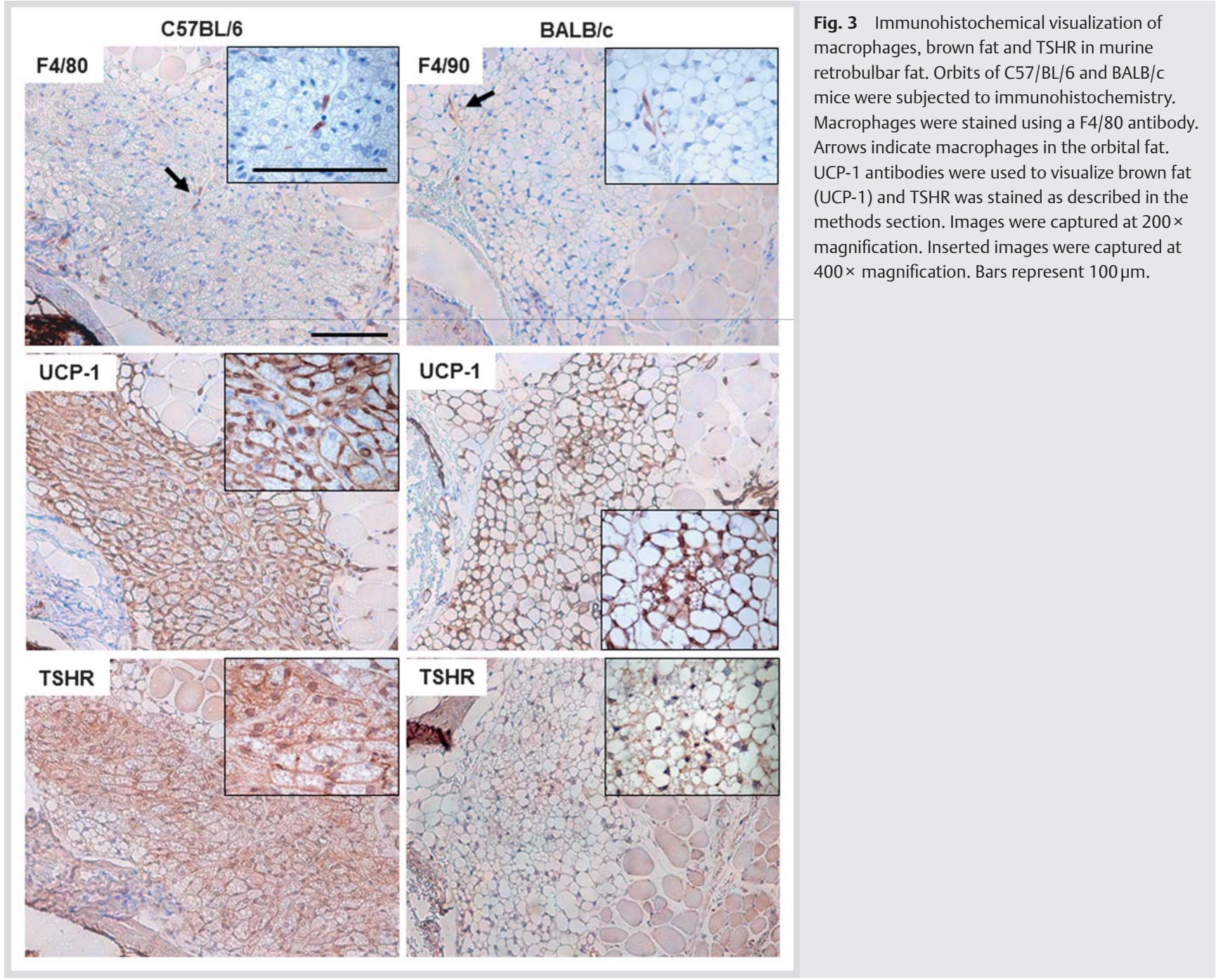

ers but did not result in significant inflammatory and proliferative orbital changes.

Many et al. were the first to report signs of orbital involvement in a mouse model of GD [22]. Attempts by members of the same group to re-establish this model in Cardiff failed, water imported even with drinking water, imported from Brussels, as well as identical chow and bedding and mice obtained from the same distributor. In the end it was concluded that differences in microbial flora had rendered the animals resistant to induced GD [23,32]. The authors described deposits of an amorphous material or fibrous tissue and adipose tissue infiltrating the extraocular muscles, often in association with mast cells and macrophages. However, the described orbital changes were difficult to quantify. Our technique of in toto removal of the mouse orbit allows significantly improved orbital preparation. Clear anatomical landmarks are visible. The presented technique will increase the sensitivity of detection because artefacts can be easily revealed.

We further examined the occurrence of brown adipose tissue (specific marker UCP-1) in murine orbits. Brown adipose tissue is involved in thermogenesis and expresses high levels of the TSHR [33] - the target antigen in Graves' orbitopathy. UCP-1 has been detected in human orbital preadipocytes [34], which are involved in pathologically increased adipogenesis in GO. Our immunohistological findings revealed brown adipose tissue in all examined mouse strains and abundant expression of TSHR. We did not examine the effect of immunization on UCP-1 staining. We did also not quantify the UCP-1 and TSHR content in different strains since both receptors were abundantly expressed. We selected BALB/c and C57BL/6 due to the fact that those strains were most often used to successfully establish mouse models in GD and various knock outs of these strains are available.

In most studies orbital changes have not been examined systematically $[8-17,19,20,25]$ and groups who did examine the orbit had not applied our approach of full orbital removal, so subtle changes in the orbit might have been overlooked $[14,16]$. We therefore examined mouse orbits after immunization with a plasmid coding for the TSHR activating mutant D633H or a TSHR A-subunit adenovirus, which are techniques that have reliably induced thyroid autoimmunity before $[17,25]$.

The prevalence of TSAb varies slightly throughout the literature. Immunization of Swiss outbred mice using the plasmid encoding for the activating mutant $\mathrm{D} 633 \mathrm{H}$ ended up with $90 \%$ prevalence of stimulating TSAb [25]. Although a similar immunization protocol was used, we detected the stimulating TSAb only in 2/9 $\mathrm{C} 57 \mathrm{BL} / 6$ mice and in none of tvhe BALB/c mice. In contrast, $\mathrm{BALB} / \mathrm{c}$ developed TSAb when immunized by in vivo electroporation with the TSHR A-subunit plasmid alone or in combination with an IGF-1R $\alpha$ encoding plasmid [35]. Some of those mice also developed pathological changes in thyroid (42\% vs. $33 \%$ ) and 

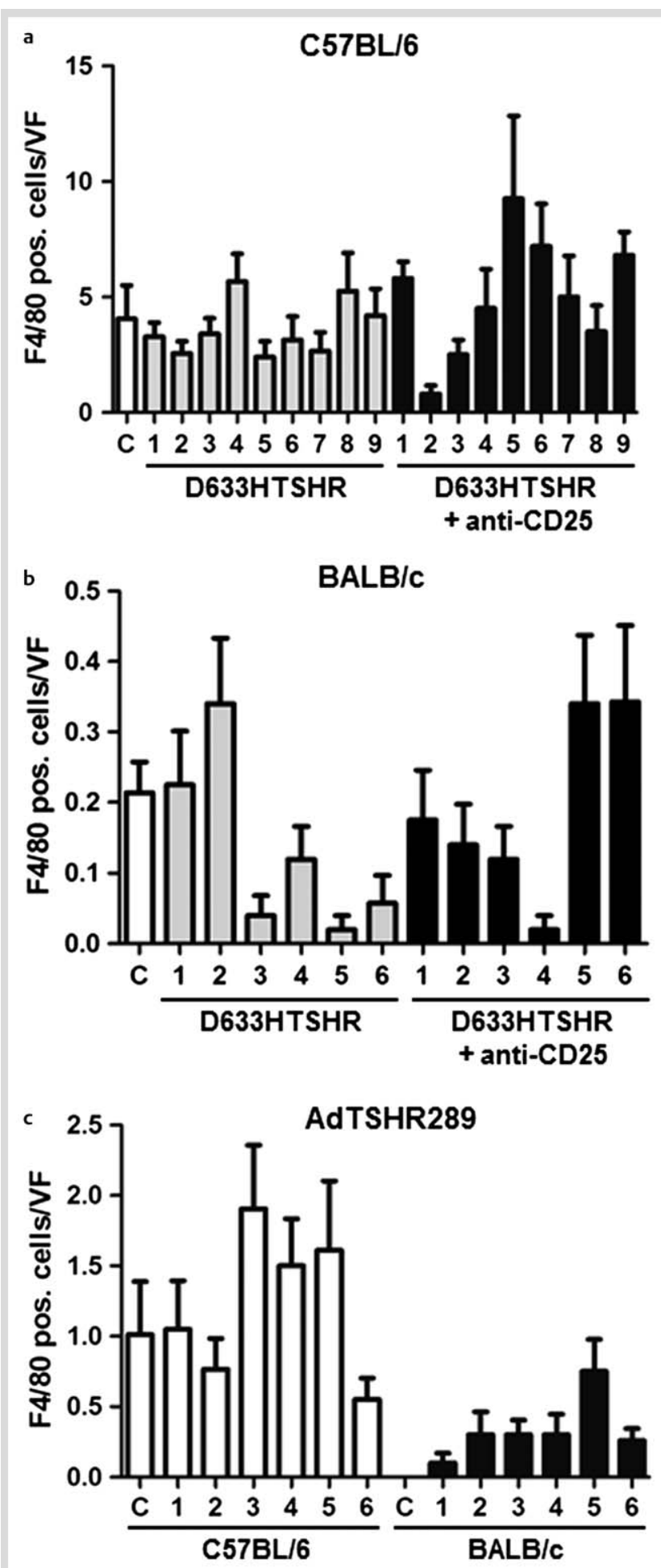

Fig. 4 Analysis of F4/80 immunohistochemical staining of mouse orbits. Orbits of immunized C57BL/6 or BALB/c mice were subjected to immunohistochemistry using a F4/80 antibody to visualize macrophages. F4/80 positive cell counts/visual field (VF) were established blindly in duplicate at $400 \times$ magnification, as visualized in Fig. 4a. a C57BL/6 mice were immunized with $4 \times$ D633HTSHR with or without CD25 depletion (mouse no. 1-9). b BALB/c mice immunized with $4 \times$ D633HTSHR with or without CD25 depletion (mouse no. 1-6). c C57BL/6 and BALB/C mice immunized with $2 \times$ AdTSHR289 (mouse number $1-6$ of immunized mice). The data are the means of at least 5 visual fields in 4 slides per animal. The error bars represent standard error. c Control mice of the respective mouse strain. The data are the means of $\mathrm{F} / 480$ pos. cells/visual field of 3 control mice. The error bars represent standard error. orbit (both groups $83 \%$ ). Histological analysis of the orbital tissues revealed moderate connective tissue fibrosis and deposition of Masson's trichrome staining material [35].

Depletion of CD25 + regulatory T cells with an anti-CD25 antibody leads to a significantly higher incidence of stimulating antibodies in C57BL/6 and to more marked hyperthyroidism in $\mathrm{BALB} / \mathrm{c}$ mice after adenoviral immunization [17]. Likewise, when we used the D633HTSHR plasmid in combination with CD25 depletion, a higher percentage of C57BL/6 mice (4/9) showed TSAb. However, none of the BALB/c mice were TSAb positive.

In contrast, regardless of strain, almost all AdTSHR289 immunized mice developed TSAb (6/6 C57BL/6 and 5/6 of the BALB/C mice). This confirms the results of Saitoh et al. [17] who found that C57BL/6 and BALB/C developed TSAb when immunized with AdTSHR289, but only BALB/c mice develop hyperthyroidism. Depletion of CD25 rendered some C57BL/6 mice susceptible to hyperthyroidism, which also developed extremely high TSAb activities [17]. Plasmid or adenoviral immunization with the TSHR A-subunit induces TSAb in C57BL/6 and BALB/c mice, whereas immunization with the D633HTSHR plasmid was less efficient.

Although mice immunized with the D633HTSHR plasmid alone did not develop TSAb within the first weeks after immunization, among CD8 + splenocytes there was a significant portion of specific anti-TSHR reacting T-cells. The D633H mutant is more intensely expressed on the cell surface than other gain of function mutants [36], which allows antigen recognition by the immune system. However, despite the TSHR epitope-specific cellular immune response, humoral immunity could not be induced at all with the shorter immunization protocols. Nevertheless, it is noteworthy that TSHR specific CD8 + T cells were detected after immunization with the D633HTSHR plasmid, indicating for the first time the participation of target specific cellular immune response in a murine model of Graves.

We did not detect significant infiltration of orbital tissues with macrophages or lymphocytes, nor did we see changes of the extracellular matrix in any of the immunized animals. The most obvious explanation, according to recently published literature, seems to be the difficulty to break tolerance to the TSHR and possible cofactors, which are necessary to induce orbitopathy.

Recently, Nakahara et al. [37] described an elegant method to induce genuine TSHR autoimmunity in mice: Splenocytes from TSHR knockout BALB/c mice, which had been immunized with adenovirus, expressing the mTSHR A-subunit, were transferred into female athymic mice. Anti-TSHR autoantibodies were detected in approximately $50 \%$ of recipient mice 4 weeks after adoptive transfer of splenocytes. Our group had the opportunity to examine the orbits of 9 of those mice, using our new method. In 2 of those mice we detected significantly increased numbers of infiltrating macrophages in both, the adipose tissues and the interstitium of muscle tissues. This clearly indicates a major role of the TSHR in the pathogenesis of Graves' orbitopathy and hints that more efficient ways to overcome tolerance to the TSHR are necessary to induce orbital changes.

In conclusion, we have established a reliable technique to study manifestations of orbitopathy in mouse models of Graves' disease. We showed that common immunization protocols lead to production of TSAb, but do not necessarily result in significant pathological changes in the orbit of $\mathrm{C} 57 \mathrm{BL} / 6$ or $\mathrm{BALB} / \mathrm{c}$ mice. It appears that either immunization against the TSHR A subunit and/or IGF1R by in vivo electroporation [35], or adoptive transfer of primed splenocytes from TSHR knockout mice [37] are necessary to evoke the full spectrum of Graves' disease in mice. 
Yet even in those animals, orbital changes were still subtle, suggesting that further modifications will be necessary to increase disease severity, particularly with regard to orbital pathology.

\section{Acknowledgements}

This project was supported by the IFORES program of the University Duisburg-Essen.

\section{Conflict of Interest}

The authors declare that they have no conflicts of interest in the authorship or publication of this contribution.

\section{Affiliations}

'Department for Ophthalmology, University of Duisburg-Essen, Essen, Germany ${ }^{2}$ Department of Endocrinology and Diabetology, University of Düsseldorf, Düsseldorf, Germany

${ }^{3}$ Institute for Experimental Endocrinology - EnForCé, Charité, Berlin, Germany ${ }^{4}$ Department of Molecular Medicine, Atomic Bomb Disease Institute, Japan University Graduate School of Biomedical Sciences, Nagasaki, Japan

${ }^{5}$ Department of Molecular Biology, University of Duisburg-Essen, Essen, Germany

\section{References}

1 Eckstein AK, Johnson KT, Thanos M, Esser J, Ludgate M. Current insights into the pathogenesis of Graves' orbitopathy. Horm Metab Res 2009; 41: 456-464

2 Prabhakar BS, Bahn RS, Smith TJ. Current perspective on the pathogenesis of Graves' disease and ophthalmopathy. Endocr Rev 2003; 24: 802-835

3 Bahn RS. Clinical review 157: Pathophysiology of Graves' ophthalmopathy: the cycle of disease. J Clin Endocrinol Metab 2003; 88: 1939-1946

4 Khoo DH, Ho SC, Seah LL, Fong KS, Tai ES, Chee SP, Eng PH, Aw SE, Fok $A C$. The combination of absent thyroid peroxidase antibodies and high thyroid-stimulating immunoglobulin levels in Graves' disease identifies a group at markedly increased risk of ophthalmopathy. Thyroid 1999; 9: 1175-1180

5 Gerding MN, van der Meer JW, Broenink M, Bakker O, Wiersinga WM, Prummel MF. Association of thyrotrophin receptor antibodies with the clinical features of Graves' ophthalmopathy. Clin Endocrinol (Oxf) 2000; 52: 267-271

6 Noh JY, Hamada N, Inoue Y, Abe Y, Ito K. Thyroid-stimulating antibody is related to Graves' ophthalmopathy, but thyrotropin-binding inhibitor immunoglobulin is related to hyperthyroidism in patients with Graves' disease. Thyroid 2000; 10: 809-813

7 Eckstein AK, Plicht M, Lax H, Neuhauser M, Mann K, Lederbogen S, Heckmann C, Esser J, Morgenthaler NG. TSH-receptor autoantibodies are independent risk factors for Graves' ophthalmopathy and help to predict severity and outcome of the disease. J Clin Endocrinol Metab 2006; 91: 3464-3470

8 Kaithamana S, Fan J, Osuga Y, Liang SG, Prabhakar BS. Induction of experimental autoimmune Graves' disease in BALB/c mice. J Immunol 1999; 163: 5157-5164

9 Shimojo N, Kohno Y, Yamaguchi K, Kikuoka S, Hoshioka A, Niimi $H_{,}$ Hirai A, Tamura Y, Saito Y, Kohn LD, Tahara K. Induction of Graves-like disease in mice by immunization with fibroblasts transfected with the thyrotropin receptor and a class II molecule. Proc Natl Acad Sci USA 1996; 93: 11074-11079

10 Costagliola S, Alcalde L, Tonacchera M, Ruf J, Vassart G, Ludgate M. Induction of thyrotropin receptor (TSH-R) autoantibodies and thyroiditis in mice immunised with the recombinant TSH-R. Biochem Biophys Res Commun 1994; 199: 1027-1034

11 Costagliola S, Rodien P, Many MC, Ludgate M, Vassart G. Genetic immunization against the human thyrotropin receptor causes thyroiditis and allows production of monoclonal antibodies recognizing the native receptor. J Immunol 1998; 160: 1458-1465

12 Nagayama Y. Animal models of Graves' hyperthyroidism. Endocr J 2005; 52: 385-394

13 Nagayama Y. Graves' animal models of Graves' hyperthyroidism. Thyroid 2007; 17: 981-988

14 Kita-Furuyama M, Nagayama Y, Pichurin P, McLachlan SM, Rapoport $B$, Eguchi $K$. Dendritic cells infected with adenovirus expressing the thyrotrophin receptor induce Graves' hyperthyroidism in BALB/c mice. Clin Exp Immunol 2003; 131: 234-240
15 Yamaguchi K, Shimojo N, Kikuoka S, Hoshioka A, Hirai A, Tahara K, Kohn $L D$, Kohno Y, Niimi H. Genetic control of anti-thyrotropin receptor antibody generation in $\mathrm{H}-2 \mathrm{~K}$ mice immunized with thyrotropin receptortransfected fibroblasts. J Clin Endocrinol Metab 1997; 82: 4266-4269

16 Nagayama Y, Kita-Furuyama M, Ando T, Nakao K, Mizuguchi H, Hayakawa T, Eguchi K, Niwa M. A novel murine model of Graves' hyperthyroidism with intramuscular injection of adenovirus expressing the thyrotropin receptor. J Immunol 2002; 168: 2789-2794

17 Saitoh 0 , Nagayama Y. Regulation of Graves' hyperthyroidism with naturally occurring $C D 4+C D 25$ + regulatory T cells in a mouse model. Endocrinology 2006; 147: 2417-2422

18 Patibandla SA, Fan JL, Prabhakar BS, Seetharamaiah GS. Comparison of immune responses to extracellular domains of mouse and human thyrotropin receptor. J Autoimmun 1999; 13: 205-213

19 Land KJ, Gudapati P, Kaplan MH, Seetharamaiah GS. Differential requirement of signal transducer and activator of transcription-4 (Stat4) and Stat6 in a thyrotropin receptor-289-adenovirus-induced model of Graves' hyperthyroidism. Endocrinology 2006; 147: 111-119

20 Seetharamaiah GS, Land KJ. Differential Susceptibility of BALB/c and BALB/cBy mice to Graves' hyperthyroidism. Thyroid 2006; 16: 651-658

21 Kaneda T, Honda A, Hakozaki A, Fuse T, Muto A, Yoshida T. An improved Graves' disease model established by using in vivo electroporation exhibited long-term immunity to hyperthyroidism in BALB/c mice. Endocrinology 2007; 148: 2335-2344

22 Many MC, Costagliola S, Detrait M, Denef F, Vassart G, Ludgate MC. Development of an animal model of autoimmune thyroid eye disease. J Immunol 1999; 162: 4966-4974

23 Ludgate M, Baker G. Inducing Graves' ophthalmopathy. J Endocrinol Invest 2004; 27: 211-215

24 Ludgate ME. Animal models of thyroid-associated ophthalmopathy. Thyroid 2002; 12: 205-208

25 Ho SC, Goh SS, Kee IH, Chow PK, Yeo CP, Khoo DH. Effects of genetic immunization of Swiss outbred mice with human thyroid stimulating hormone receptor cDNA plasmids harboring gain-of-function mutations. J Mol Endocrinol 2007; 38: 277-288

26 Ulmer JB, DeWitt CM, Chastain M, Friedman A, Donnelly JJ, McClements WL, Caulfield MJ, Bohannon KE, Volkin DB, Evans RK. Enhancement of DNA vaccine potency using conventional aluminum adjuvants. Vaccine 1999; 18: 18-28

27 Andrew SM, Titus JA. Purification of immunoglobulin G. Curr Protoc Immunol 2001 Chapter 2: Unit 27

28 Rammensee H, Bachmann J, Emmerich NP, Bachor OA, Stevanovic S. SYFPEITHI: database for MHC ligands and peptide motifs. Immunogenetics 1999; 50: 213-219

29 Morgenthaler NG, Ho SC, Minich WB. Stimulating and blocking thyroidstimulating hormone (TSH) receptor autoantibodies from patients with Graves' disease and autoimmune hypothyroidism have very similar concentration, TSH receptor affinity, and binding sites. J Clin Endocrinol Metab 2007; 92: 1058-1065

30 Mark M, Teletin M, Antal C, Wendling O, Auwerx J, Heikkinen S, Khetchoumian K, Argmann CA, Dgheem M. Histopathology in mouse metabolic investigations. In: Ausubel FM, Brent R, Kingston RE, Moore DD, Seidman JG, Smith JA, Struhl K (eds.). Current Protocols in Molecular Biology. Hoboken, N. J.: Wiley, 2007 Chapter 29: Unit 29B 24

31 Payne AP. The harderian gland: a tercentennial review. Journal of anatomy 1994; 185 (Pt 1): 1-49

32 Baker G, Mazziotti G, von Ruhland C, Ludgate M. Reevaluating thyrotropin receptor-induced mouse models of Graves' disease and ophthalmopathy. Endocrinology 2005; 146: 835-844

33 Endo T, Kobayashi T. Thyroid-stimulating hormone receptor in brown adipose tissue is involved in the regulation of thermogenesis. American journal of physiology Endocrinology and metabolism 2008; 295: E514-E518

34 Zhang L, Baker G, Janus D, Paddon CA, Fuhrer D, Ludgate M. Biological effects of thyrotropin receptor activation on human orbital preadipocytes. Invest Ophthalmol Vis Sci 2006; 47: 5197-5203

35 Zhao SX, Tsui S, Cheung A, Douglas RS, Smith TJ, Banga JP. Orbital fibrosis in a mouse model of Graves' disease induced by genetic immunization of thyrotropin receptor cDNA. J Endocrinol 2011; 210: 369-377

36 Ho SC, Goh SS, Su Q, Khoo DH. Cysteine 390 mutation of the TSH receptor modulates its ectodomain as an inverse agonist on the serpentine domain with decrease in basal constitutive activity. Mol Cell Endocrinol 2005; 245: 158-168

37 Nakahara M, Johnson K, Eckstein A, Taguchi R, Yamada M, Abiru N, Nagayama $Y$. Adoptive transfer of antithyrotropin receptor (TSHR) autoimmunity from TSHR knockout mice to athymic nude mice. Endocrinology 2012; 153: 2034-2042 